\title{
Dimensão da malha amostral para caracterização da variabilidade espacial de fósforo e potássio em Latossolo Vermelho
}

\author{
Maurício Roberto Cherubin ${ }^{(1)}$, Antônio Luis Santi(2), Mateus Tonini Eitelwein(1), Telmo Jorge Carneiro Amado ${ }^{(3)}$, \\ Diego Henrique Simon ${ }^{(2)}$ e Júnior Melo Damian(2)
}

\begin{abstract}
(1)Escola Superior de Agricultura Luiz de Queiroz, Universidade de São Paulo, Avenida Pádua Dias, no 11, CEP 13418-900 Piracicaba, SP, Brasil. E-mail: cherubin@usp.br, mateus_eitelwein@usp.br(2)Universidade Federal de Santa Maria, Centro de Educação Superior Norte do Rio Grande do Sul, Linha Sete de Setembro, s/no, BR 386, Km 40, CEP 98400-000 Frederico Westphalen, RS, Brasil. E-mail: santi_pratica@yahoo.com.br, tecsimon@hotmail.com, juniormelodamian@hotmail.com ${ }^{(3)}$ Universidade Federal de Santa Maria, Centro de Ciências Rurais, CEP 97105-900 Santa Maria, RS, Brasil. E-mail: telmo.amado@pq.cnpq.br
\end{abstract}

Resumo - O objetivo deste trabalho foi avaliar a acurácia na caracterização da variabilidade espacial de fósforo e potássio disponíveis no solo, pelo uso de diferentes dimensões de malhas amostrais, bem como a similaridade dos mapas temáticos gerados. O estudo foi conduzido em área de Latossolo Vermelho de 41,96 ha, em Boa Vista das Missões, RS. A amostragem de solo foi realizada na camada de 0,00-0,10 m, tendo-se utilizado sete

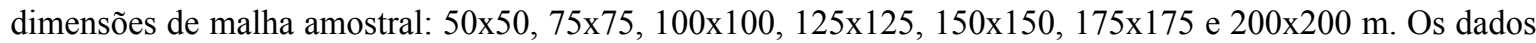
de $\mathrm{P}$ e $\mathrm{K}$ foram submetidos às análises de estatística descritiva e de geoestatística, e a similaridade dos mapas temáticos gerados foi analisada pelo coeficiente de desvio relativo e pela matriz de correlação de Pearson. A redução da dimensão da malha amostral aumenta a acurácia na caracterização da variabilidade espacial de $\mathrm{P}$ e $\mathrm{K}$ e, consequentemente, a qualidade das informações geradas por meio dos mapas temáticos. Malhas amostrais $\leq 100 \times 100 \mathrm{~m}$ são recomendadas para planos de amostragem de solo adotados nas áreas de agricultura de precisão no Estado do Rio Grande do Sul.

Termos para indexação: adubação à taxa variada, agricultura de precisão, amostragem georreferenciada de solo, fertilidade do solo, similaridade de mapas temáticos.

\section{Sampling grid size for characterization of the spatial variability of phosphorus and potassium in an Oxisol}

\begin{abstract}
The objective of this work was to evaluate the accuracy in the characterization of the spatial variability of soil phosphorus and potassium, using different sampling grid sizes, as well as the similarity of the thematic maps generated. The study was carried out in a 41.96 ha Oxisol area in the municipality of Boa Vista das Missões, in the state of Rio Grande do Sul, Brazil. Soil sampling was done at the 0.00-0.10 m layer,

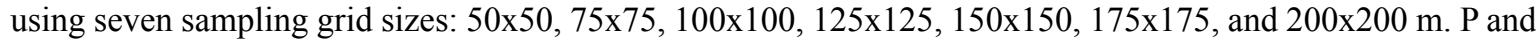
$\mathrm{K}$ data were subjected to descriptive statistics and geostatistical analyses, and the similarity of the thematic maps generated was analyzed by the coefficient of relative deviation and Pearson's correlation matrix. The reduction in the size of the sampling grid increases the accuracy in the characterization of the spatial variability of $\mathrm{P}$ and $\mathrm{K}$ and, consequently, the information generated by the thematic maps. Sampling grid sizes $\leq 100 \times 100$ $\mathrm{m}$ are recommended for soil sampling plans adopted in precision agriculture areas in the state of Rio Grande do Sul, Brazil.
\end{abstract}

Index terms: variable-rate fertilization, precision agriculture, georeferenced soil sampling, soil fertility, similarity of thematic maps.

\section{Introdução}

A agricultura de precisão (AP) é um avanço tecnológico relativamente recente de gerenciamento do sistema solo-planta-atmosfera, baseada nos princípios da caracterização da variabilidade espacial e da gestão de informações, que engloba fatores de produção e produtividade das culturas (Montanari et al., 2012). Entre as ferramentas da AP, a amostragem georreferenciada de solo por meio de malhas regulares, para caracterizar a variabilidade dos atributos químicos do solo, é uma das mais importantes e tradicionalmente utilizadas na agricultura brasileira. 
No entanto, a amostragem georreferenciada de solo, mesmo utilizada em grande escala, ainda carece de definições metodológicas, especialmente quanto à dimensão da malha amostral (Nanni et al., 2011; Montanari et al., 2012; Stepien et al., 2013; Cherubin et al., 2014; Souza et al., 2014). Na região Sul do Brasil, a Comissão de Química e Fertilidade do Solo - RS/SC (Manual de adubação e de calagem para os estados do Rio Grande do Sul e Santa Catarina, 2004) recomenda apenas que a amostragem de solo para a AP deve preconizar um número de amostras coletadas muito maior do que a amostragem tradicional. Contudo, os critérios para a definição da dimensão das malhas não foram estabelecidos até o momento.

A eficiência de um plano ou arranjo amostral de solo é dependente do conhecimento prévio da estrutura da variabilidade espaço-temporal que os atributos investigados apresentam no solo (Mallarino \& Wittry, 2004; Nanni et al., 2011; Montanari et al., 2012). Entretanto, a variabilidade espaço-temporal dos atributos do solo, resultante da formação do solo e de intervenções antrópicas, varia em diferentes escalas espaciais e temporais (Cambardella et al., 1994).

Assim, as diferentes escalas de variação dos atributos do solo dificultam o desenvolvimento de um plano de amostragem que utilize uma malha amostral com espaçamento único, quando vários atributos do solo estão envolvidos (Montanari et al., 2012; Stepien et al., 2013). Isso justifica investigações que visam definir o tamanho ideal da malha amostral para atributos de solo específicos, especialmente aqueles, como o $\mathrm{P}$ e o $\mathrm{K}$, que são os elementos mais importantes em modelos de prescrição de manejo sítio-específico no Brasil (Corá \& Beraldo 2006; Nanni et al., 2011; Cherubin et al., 2014).

Discussões referentes à dimensão da malha amostral para a caracterização da variabilidade espacial de $\mathrm{P}$ e $\mathrm{K}$ no solo têm sido relatadas na literatura. Nos Estados Unidos, estudos apontam malhas adequadas que variam de: menos que $30 \mathrm{~m}$ (Stamper et al., 2014), 45 a 125 m (Mallarino \& Wittry, 2004) e 160 a 253 m (Magri et al., 2005). Na Polônia, Stepien et al. (2013) constataram que o uso de malhas amostrais de $100 \mathrm{~m}$ possibilita caracterizar a variabilidade com maior acurácia, mas, que, dependendo do nutriente, malhas maiores, de até $200 \mathrm{~m}$, podem ser utilizadas. Para as condições brasileiras, são poucos os trabalhos que buscam definir uma dimensão ideal de malha amostral para a coleta de solo em áreas de AP. Nanni et al. (2011) e Souza et al. (2014) verificaram que a malha de $100 \mathrm{~m}$ não foi suficiente para caracterizar eficientemente a variabilidade espacial dos teores de $\mathrm{P}$ e $\mathrm{K}$, o que mostra que uma amostragem mais densa é necessária para recomendações de fertilizantes à taxa variada. Souza et al. (2014) sugerem dimensões de malhas amostrais que condicionem no mínimo 100 pontos de amostragem. Corá \& Beraldo (2006) e Montanari et al. (2012) também observaram que o uso de malhas de 100 e $200 \mathrm{~m}$, respectivamente, não foi eficiente para expressar a variabilidade espacial dos teores de $\mathrm{P}$ no solo. Cherubin et al. (2014) relataram que malhas amostrais de 100, 142 e 173 m possibilitaram captar as diferentes escalas da variabilidade espacial de $\mathrm{P}$ e $\mathrm{K}$ no solo; porém, os autores alertam que a utilização de malhas que condicionem poucos pontos de amostragem deve ser evitada, para garantir a acurácia da caracterização e o manejo da variabilidade espacial desses nutrientes.

O objetivo deste trabalho foi avaliar a acurácia na caracterização da variabilidade espacial de $\mathrm{P}$ e K disponíveis no solo, por meio de diferentes dimensões de malhas amostrais, bem como a similaridade dos mapas temáticos gerados.

\section{Material e Métodos}

$\mathrm{O}$ estudo foi conduzido em área experimental localizada no Município de Boa Vista das Missões, RS $\left(27^{\circ} 71^{\prime} 66^{\prime \prime} \mathrm{S}\right.$ a $27^{\circ} 72^{\prime} 55^{\prime \prime} \mathrm{S}$ e $53^{\circ} 33^{\prime} 13^{\prime \prime} \mathrm{W}$ a $\left.53^{\circ} 34^{\prime} 08^{\prime \prime} \mathrm{W}\right)$, o que totalizou uma área de 41,96 ha. O clima da região, de acordo com a classificação de Maluf (2000), é subtropical com primavera úmida (ST PU), com temperatura média anual de $18,1^{\circ} \mathrm{C}$ e precipitação pluvial anual de $1.919 \mathrm{~mm}$.

O relevo da região é suave ondulado, e o solo presente na área experimental foi classificado como Latossolo Vermelho distrófico típico (Santos et al., 2013), com textura muito argilosa. A área vem sendo manejada sob sistema plantio direto (SPD) há mais de dez anos, com cultivos rotacionados de soja [Glycine max (L.) Merr.] e milho (Zea mays L.) nas safras de verão e de aveia-branca (Avena sativa L.) (grãos) e aveia-preta (Avena strigosa Schreb.) (cobertura do solo) nas safras de inverno. O histórico de adubação da área é baseado em aplicações de fertilizante fosfatado 
incorporado na linha de semeadura e em aplicações superficiais de fertilizantes potássicos a lanço.

Inicialmente, realizou-se o georreferenciamento (vetorização) da área experimental a partir da demarcação do seu perímetro, tendo-se utilizado aparelho de GPS de navegação portátil. Posteriormente, sete malhas amostrais quadriculares regulares foram geradas e sobrepostas na área, cujas dimensões foram: $50 \times 50 \mathrm{~m}(0,25 \mathrm{ha}), 75 \times 75 \mathrm{~m}(0,56 \mathrm{ha}), 100 \times 100 \mathrm{~m}$ (1,0 ha), 125x125 m (1,56 ha), 150x150 m (2,25 ha), $175 \times 175 \mathrm{~m}$ (3,06 ha) e 200x200 m (4,0 ha). No centro de cada quadrícula, foram alocados os pontos georreferenciados de coleta de solo, o que totalizou $164,74,38,26,16,14$ e 9 pontos para as respectivas malhas amostrais. As malhas amostrais foram geradas por meio do programa computacional CR Campeiro 7 (Departamento de Geomática, Universidade Federal de Santa Maria, RS).

A coleta de solo foi realizada em todas as malhas amostrais, tendo-se utilizado trado calador de forma manual. Cada amostra foi composta por dez subamostras, coletadas num raio de aproximadamente $10 \mathrm{~m}$ do ponto georreferenciado, na profundidade de 0,00-0,10 m, recomendada pela Comissão de Química e Fertilidade do Solo - RS/SC (Manual de adubação e de calagem para os estados do Rio Grande do Sul e Santa Catarina, 2004) em áreas de SPD consolidado. As amostras foram encaminhadas ao laboratório, e os teores de $\mathrm{P}$ e $\mathrm{K}$ disponíveis foram determinados pelo método de extração Mehlich-1 (Manual de adubação e de calagem para os estados do Rio Grande do Sul e Santa Catarina, 2004). Em relação aos parâmetros interpretativos de $\mathrm{P}$ e $\mathrm{K}$ disponíveis no solo, constatouse que a área apresentava, em média, altos teores de argila $(>60 \%)$ e $\mathrm{CTC}_{\mathrm{pH} 7}$ média de $11,3 \mathrm{cmol}_{\mathrm{c}} \mathrm{dm}^{-3}$.

Os dados de $\mathrm{P}$ e $\mathrm{K}$ foram submetidos à análise estatística descritiva, para verificar a posição e a dispersão dos dados, com o auxílio do programa computacional SAS, versão 8.0 (SAS Institute, Cary, NC, USA). Os parâmetros estatísticos determinados foram: mínimo; média; mediana; máximo; desvio-padrão; erro-padrão; e coeficientes de variação $(\mathrm{CV})$, de precisão (CP), de assimetria (Cs) e de curtose (Ck). Com base nos valores de CV obtidos, a dispersão dos dados foi classificada em: baixa, para $\mathrm{CV}<15 \%$; moderada, para CV de 15 a $35 \%$; e alta, para CV $>35 \%$ (Wilding \& Drees, 1983). A tendência central (normalidade) dos dados originais foi testada por meio do teste de Shapiro-Wilk, a 5\% de probabilidade.

A análise da variabilidade espacial de $\mathrm{P}$ e $\mathrm{K}$ foi processada por meio de semivariogramas, cujos ajustes foram realizados por modelos matemáticos teóricos, com uso do conjunto de programas computacionais Geoest (Vieira et al., 2002). O ajuste dos modelos foi realizado com base no melhor coeficiente de determinação $\left(\mathrm{R}^{2}\right)$ e na menor soma de quadrados do resíduo (SQR), e foi confirmado pela técnica de validação cruzada. Do ajuste de um modelo matemático aos dados, foram definidos os seguintes parâmetros: efeito pepita $\left(\mathrm{C}_{0}\right)$, contribuição $\left(\mathrm{C}_{1}\right)$, patamar $\left(\mathrm{C}_{0}+\mathrm{C}_{1}\right)$ e alcance $(\mathrm{a})$. $\mathrm{O}$ índice de dependência espacial (IDE) foi calculado por meio da equação: IDE $=\left[\mathrm{C}_{0} /\left(\mathrm{C}_{0}+\mathrm{C}_{1}\right)\right] \times 100$. Com base nos IDE, classificou-se o grau de dependência espacial (GDE) como: forte, para IDE $\leq 25 \%$; moderado, para IDE entre 25 e $75 \%$; e fraco, para IDE $>75 \%$ (Cambardella et al., 1994).

Para a construção dos mapas temáticos, foram utilizados os métodos de interpolação por krigagem, para os dados que apresentaram estrutura espacial, e do inverso do quadrado da distância, para os dados que não apresentaram estrutura espacial (efeito pepita puro). Os mapas temáticos foram gerados com o auxílio do programa computacional CR Campeiro 7 (Departamento de Geomática, Universidade Federal de Santa Maria, RS).

A similaridade dos mapas temáticos de $\mathrm{P}$ e $\mathrm{K}$, construídos a partir dos dados coletados nas diferentes malhas amostrais, foi avaliada por meio de dois parâmetros estatísticos: o coeficiente de desvio relativo $(\mathrm{CDR}, \%)$ e a matriz de correlação linear simples de Pearson $(\mathrm{p}<0,05)$. O CDR expressa a diferença média, em módulo, dos valores interpolados em cada mapa e considera um deles como mapa referência (padrão). Quanto menor for o percentual encontrado, maior será a similaridade entre os mapas. O cálculo é dado pela equação adaptada de Coelho et al. (2009): $\mathrm{CDR}=\sum \mid($ Tij - Tiref $) /$ Tiref $\mid \times(100 / \mathrm{n})$, em que: $n$ é o número de pontos interpolados $(n=164$ pontos); Tiref é o teor referência do nutriente no ponto i, sendo que os teores de referência do nutriente foram obtidos na malha mais densa de 50x50 m; e Tij é o teor do nutriente no ponto i para as diferentes dimensões de malhas amostrais $\mathrm{j}(\mathrm{j} 1,50 \times 50 \mathrm{~m} ; \mathrm{j} 2,75 \times 75 \mathrm{~m}$; 
j3, 100x100 m; j4, 125x125 m; j5, 150x150 m; j6, 175x175 m; e j7, 200x200 m).

Para realizar a matriz de correlação linear simples de Pearson $(p<0,05)$, igualou-se o conjunto de dados (n) para todos os mapas temáticos gerados a partir das malhas estudadas. Para tanto, do mesmo modo que no cálculo do CDR, foram utilizados os valores dos 164 pontos amostrais no mapa cuja malha era mais densa $(50 \times 50 \mathrm{~m})$ e os valores interpolados nesses mesmos 164 locais, nos demais mapas.

\section{Resultados e Discussão}

Os resultados da análise estatística descritiva são indicativos de que o solo estudado apresenta alta fertilidade (Tabela 1), com teores médios de P de 16,4 a $26,5 \mathrm{mg} \mathrm{dm}^{-3}$ e de $\mathrm{K}$ de 220,6 a $253,9 \mathrm{mg} \mathrm{dm}^{-3}$, ambos acima do nível crítico $\left(\mathrm{P}>6 \mathrm{mg} \mathrm{dm}^{-3}\right.$ e $\left.\mathrm{K}>60 \mathrm{mg} \mathrm{dm}^{-3}\right)$, considerados satisfatórios para o desenvolvimento das culturas (Manual de adubação e de calagem para os estados do Rio Grande do Sul e Santa Catarina, 2004).

À medida que a dimensão da malha amostral aumentou, houve redução na amplitude entre os valores mínimos e máximos obtidos, tendendo para uma aproximação aos valores médios. Assim, o aumento no número de amostras coletadas (n), proporcionado por malhas amostrais menores, possibilita identificar locais da área onde os teores de $\mathrm{P}$ e $\mathrm{K}$ são extremos e, se não corrigidos, podem induzir à ocorrência de zonas de restrição à produtividade das culturas.

Em relação às medidas de posição central, observouse que os valores das médias e das medianas foram

Tabela 1. Estatísticas descritivas dos teores de fósforo e potássio determinados com uso de diferentes dimensões de malhas amostrais em área de Latossolo Vermelho no Sul do Brasil.

\begin{tabular}{|c|c|c|c|c|c|c|c|}
\hline \multirow[t]{2}{*}{ Parâmetros estatísticos $^{(1)}$} & \multicolumn{7}{|c|}{ Malha amostral (m) } \\
\hline & $50 \times 50$ & $75 \times 75$ & $100 \times 100$ & $125 \times 125$ & $150 \times 150$ & $175 \times 150$ & $200 \times 200$ \\
\hline & \multicolumn{7}{|c|}{ Fósforo (mg dm$\left.{ }^{-3}\right)$} \\
\hline $\mathrm{n}$ & 164 & 74 & 38 & 26 & 16 & 14 & 9 \\
\hline Mínimo & 5,50 & 6,56 & 8,40 & 11,30 & 14,30 & 12,40 & 11,70 \\
\hline Média & 25,30 & 25,60 & 22,88 & 26,46 & 26,09 & 25,47 & 16,44 \\
\hline Mediana & 23,90 & 23,84 & 22,10 & 25,80 & 24,15 & 25,05 & 16,60 \\
\hline Máximo & 54,90 & 54,70 & 51,20 & 41,00 & 52,70 & 39,80 & 22,90 \\
\hline Desvio-padrão & 8,80 & 9,94 & 9,03 & 7,41 & 10,46 & 7,49 & 3,60 \\
\hline Erro-padrão (\%) & 5,34 & 8,91 & 12,71 & 10,97 & 20,28 & 15,98 & 15,16 \\
\hline $\mathrm{CV}(\%)$ & 34,79 & 38,84 & 39,45 & 27,98 & 40,08 & 29,39 & 21,87 \\
\hline $\mathrm{CP}(\%)$ & 2,72 & 4,52 & 6,40 & 5,49 & 10,02 & 7,85 & 7,29 \\
\hline Cs & 0,73 & 0,29 & 0,70 & 0,39 & 1,35 & 0,07 & 0,30 \\
\hline $\mathrm{Ck}$ & 0,80 & $-0,37$ & 1,05 & $-0,21$ & 1,79 & $-0,22$ & $-0,05$ \\
\hline \multirow[t]{2}{*}{ Teste de Shapiro-Wilk } & $0,97^{\mathrm{ns}}$ & $0,98^{\text {ns }}$ & $0,96^{\mathrm{ns}}$ & $0,95^{\mathrm{ns}}$ & $0,87 *$ & $0,98^{\text {ns }}$ & $0,96^{\mathrm{ns}}$ \\
\hline & \multicolumn{7}{|c|}{ Potássio $\left(\mathrm{mg} \mathrm{dm}^{-3}\right)$} \\
\hline $\mathrm{n}$ & 164 & 74 & 38 & 26 & 16 & 14 & 9 \\
\hline Mínimo & 130,00 & 160,00 & 180,00 & 195,00 & 185,00 & 180,00 & 180,00 \\
\hline Média & 235,49 & 250,74 & 237,50 & 236,54 & 220,62 & 252,86 & 253,89 \\
\hline Mediana & 230,00 & 250,00 & 235,00 & 230,00 & 215,00 & 267,50 & 270,00 \\
\hline Máximo & 355,00 & 310,00 & 315,00 & 290,00 & 355,00 & 305,00 & 290,00 \\
\hline Desvio-padrão & 34,33 & 28,36 & 34,02 & 24,48 & 38,89 & 36,73 & 33,24 \\
\hline Erro-padrão (\%) & 2,24 & 2,60 & 4,62 & 4,06 & 8,92 & 7,90 & 9,07 \\
\hline $\mathrm{CV}(\%)$ & 14,58 & 11,31 & 14,33 & 10,35 & 17,63 & 14,52 & 13,09 \\
\hline $\mathrm{CP}(\%)$ & 1,14 & 1,31 & 2,32 & 2,03 & 4,41 & 3,88 & 4,36 \\
\hline Cs & 0,36 & $-0,16$ & 0,38 & 0,53 & 2,98 & $-0,79$ & $-1,50$ \\
\hline $\mathrm{Ck}$ & 0,84 & 0,45 & $-0,48$ & $-0,15$ & 10,66 & $-0,11$ & 2,53 \\
\hline Teste de Shapiro-Wilk & $0,98^{\mathrm{ns}}$ & $0,98^{\mathrm{ns}}$ & $0,97^{\mathrm{ns}}$ & $0,96^{\mathrm{ns}}$ & $0,65 *$ & $0,92^{\mathrm{ns}}$ & $0,86^{\mathrm{ns}}$ \\
\hline
\end{tabular}

${ }^{(1)} n$, número de observações (amostras); CV, coeficiente de variação; CP, coeficiente de precisão; Cs, coeficiente de assimetria; e Ck, coeficiente de curtose. ${ }^{n}$ Não significativo para distribuição normal. *Significativo para distribuição normal (hipótese de distribuição normal rejeitada), a $5 \%$ de probabilidade. 
similares, o que indica que os dados apresentaram distribuição de frequência normal, exceto para a malha 150x150 m. A normalidade dos dados pode ser comprovada pelos valores do Cs e do Ck próximos à zero. Mesmo não sendo uma exigência, a normalidade dos dados possibilita uma melhor predição dos valores não amostrados por meio da geoestatística (Webster \& Oliver, 2007).

Quanto à dispersão relativa dos dados, fornecida pelo $\mathrm{CV}$, constatou-se que os teores de $\mathrm{P}$ apresentaram CVs classificados de moderados (15-35\%) a altos $(>35 \%)$. Esses resultados corroboram os anteriormente encontrados em outros estudos em Latossolos (Corá \& Beraldo, 2006; Cherubin et al., 2011, 2014; Nanni et al., 2011; Santi et al., 2012). De acordo com muitos destes autores, a elevada variação dos teores de $\mathrm{P}$ decorre das sucessivas aplicações de fertilizantes na linha de semeadura, o que contribui para a manutenção ou o aumento da variabilidade espacial do solo. A dispersão dos valores de $\mathrm{K}$ foi classificada como baixa $(\mathrm{CV}<15 \%)$ (Mallarino \& Wittry, 2004; Cherubin et al., 2011, 2014; Santi et al., 2012) e pode estar relacionada à maior mobilidade do $\mathrm{K}$ no solo em relação ao $\mathrm{P}$ (Ernani et al., 2007), à elevada ciclagem de $\mathrm{K}$ dos resíduos culturais que são distribuídos uniformente sobre o solo e também à aplicação de fertilizantes potássicos a lanço, o que minimizaria a microvariabilidade espacial do elemento no solo.

Ao se comparar a dispersão dos dados entre as sete malhas amostrais, com uso do CP, verificou-se que, à medida que se reduziu a dimensão da malha amostral (maior número de pontos amostrais), as avaliações realizadas foram mais acuradas $(<\mathrm{CP} \%)$, com consequente redução na percentagem de erro amostral.

A análise da variabilidade espacial dos teores de $\mathrm{P}$ e $\mathrm{K}$ disponíveis no solo (Tabela 2) indicou que os dados coletados em malhas amostrais menores que $150 \times 150 \mathrm{~m}$ para $\mathrm{P}$ e que $125 \times 125 \mathrm{~m}$ para $\mathrm{K}$ apresentaram dependência espacial, o que possibilita a estimativa dos valores nos locais não amostrados por meio do interpolador geoestatístico krigagem. $\mathrm{O}$ modelo matemático que melhor ajustou-se aos semivariogramas foi o esférico, o que concorda com estudos anteriormente realizados com $\mathrm{P}$ e $\mathrm{K}$ (Corá \& Beraldo, 2006; Nanni et al., 2011; Cherubin et al., 2014; Souza et al., 2014).

O comportamento aleatório (efeito pepita puro) dos teores de $\mathrm{P}$ e $\mathrm{K}$, obtidos nas malhas amostrais maiores, está relacionado ao aumento da distância entre os pontos amostrais, associado à redução do número de pontos coletados. De acordo com Webster \& Oliver (2007) e Webster \& Lark (2012), a redução do conjunto de dados aumenta o intervalo de confiança, o que reduz a precisão dos modelos ajustados até tornarem-se aleatórios.

Os valores dos alcances do $\mathrm{P}$ variaram de $200 \mathrm{~m}$ (malha 50x50 m)a 479m(malha 100x100m), enquanto os do K variaram de $106 \mathrm{~m}$ (malha $75 \times 75 \mathrm{~m}$ ) a $384 \mathrm{~m}$ (malha $125 \times 125 \mathrm{~m}$ ). O alcance representa a distância limite na qual uma variável regionalizada apresenta continuidade espacial (dependência espacial). Dessa forma, variáveis amostradas a distâncias maiores que os valores de alcance apresentam distribuição aleatória, sendo independentes entre si (Webster \& Oliver, 2007).

A metade do valor do alcance tem sido proposta por Kerry \& Oliver (2004) como parâmetro balizador para a definição da distância máxima entre amostras e, por consequência, da dimensão da malha amostral em planos de amostragem futuros. Entretanto, o uso desse critério merece cautela e aplica-se com mais confiabilidade apenas à área investigada. Não se recomenda extrapolar essa estimativa para outras áreas, uma vez que os valores de alcances obtidos, para um mesmo atributo, são bastante variáveis entre áreas, por serem condicionados pela continuidade espacial da variável, pela distância entre os pontos amostrais e pelo número de amostras. Além disso, dependendo do tamanho da área e do valor do alcance, a utilização desse parâmetro pode conduzir à obtenção de poucos pontos amostrais, o que compromete a acurácia e a confiabilidade das informações geradas.

Os dados de $\mathrm{P}$ e $\mathrm{K}$ espacializados na forma de mapas temáticos (Figura 1) sugerem que a redução da dimensão da malha amostral, ou seja, o aumento da densidade amostral e a aproximação dos pontos amostrais promove maior detalhamento da distribuição espacial desses atributos na área e, portanto, melhora a acurácia das informações geradas por meio dos mapas temáticos. Desse modo, malhas amostrais mais densas são mais adequadas para a aplicação de fertilizantes à taxa variada plena; já as malhas amostrais menos densas poderiam ser utilizadas para a definição de zonas de fertilidade e a aplicação de fertilizantes à taxa variada simplificada. Essas constatações estão de acordo com os resultados de Souza et al. (2014). 
Os resultados obtidos corroboram os de Coelho et al. (2009), que inferem que, à medida que se aproximam os pontos amostrais, mais acurados são os valores dos locais não amostrados estimados por interpoladores geoestatísticos. Assim, espera-se que pontos de amostragens localizados mais próximos sejam mais parecidos entre si do que os mais afastados, o que reduziria a probabilidade de erros (Webster \& Oliver, 2007).

Verificou-se, por meio de procedimentos estatísticos e geoestatísticos, que as dimensões de malhas amostrais menores que $150 \times 150 \mathrm{~m}$ para $\mathrm{P}$ e que $125 \times 125 \mathrm{~m}$ para $\mathrm{K}$ foram capazes de captar a variabilidade espacial desses elementos no solo. A partir desse resultado, a decisão de optar por utilizar uma malha maior ou menor deve levar em consideração fatores como: histórico e situação do manejo da fertilidade do solo, tamanho da área, custo da amostragem e análise do solo, intensificação e tecnificação do sistema produtivo, nível de precisão desejado, equipamentos disponíveis para aplicação de fertilizantes, entre outros. Contudo, como alertado por Cherubin et al. (2014), sempre que possível, deve-se optar por malhas amostrais menores, uma vez que semivariogramas gerados com uso delimitado de número de pontos (n) podem comprometer a consistência das informações geradas. De acordo com Landim (2006), para a análise geoestatística, devem ser adotados no mínimo 30 pares para cada distância estudada. No presente trabalho, as malhas que possibilitaram atender a esse critério foram aquelas $\leq 100 \times 100 \mathrm{~m}$. Portanto, em áreas agrícolas com dimensão semelhante à estudada (aproximadamente $40 \mathrm{ha}$ ), sugere-se que a caracterização e o manejo da variabilidade espacial de $\mathrm{P}$ e $\mathrm{K}$ no solo sejam realizados a partir da coleta de uma ou mais amostras por hectare (malhas $\leq 100 \times 100 \mathrm{~m}$ ).

Com base nos resultados do CDR (Figura 2), constatou-se que, conforme aumentou a dimensão da malha amostral e, consequentemente, a distância entre os pontos amostrais, houve aumento da dissimilaridade dos mapas obtidos, em relação ao mapa da menor malha $(50 \times 50 \mathrm{~m})$, estabelecida como referência. Esse aumento foi ajustado por regressões lineares, com coeficientes de determinação $\left(R^{2}\right)$ de 0,91 e 0,59 para os mapas de $\mathrm{P}$ e $\mathrm{K}$, respectivamente. Os mapas temáticos de $\mathrm{P}$ apresentaram maior dissimilaridade entre si, com CDR que variou de 24 a 36,2\%, enquanto, nos mapas

Tabela 2. Parâmetros geoestatísticos dos valores de fósforo e potássio determinados com uso de diferentes dimensões de malhas amostrais em área de Latossolo Vermelho no Sul do Brasil.

\begin{tabular}{|c|c|c|c|c|c|c|c|}
\hline \multirow{2}{*}{$\begin{array}{l}\text { Parâmetros } \\
\text { geoestatísticos }^{(1)}\end{array}$} & \multicolumn{7}{|c|}{ Malha amostral (m) } \\
\hline & $50 \times 50$ & $75 \times 75$ & $100 \times 100$ & $125 \times 125$ & $150 \times 150$ & $175 \times 175$ & $200 \times 200$ \\
\hline & \multicolumn{7}{|c|}{ Fósforo } \\
\hline EPP & 17,00 & 31,00 & 3,00 & 14,00 & 1,00 & EPP & EPP \\
\hline Patamar & 82,00 & 123,00 & 96,00 & 55,00 & 137,00 & EPP & EPP \\
\hline Contribuição & 65,00 & 92,00 & 93,00 & 41,00 & 136,00 & EPP & EPP \\
\hline Alcance & 200,00 & 469,00 & 479,00 & 250,00 & 379,00 & EPP & EPP \\
\hline Modelo & Esférico & Esférico & Esférico & Esférico & Esférico & EPP & EPP \\
\hline $\mathrm{R}^{2}$ & 0,68 & 0,32 & 0,54 & 0,18 & 0,64 & EPP & EPP \\
\hline IDE & 20,73 & 25,20 & 3,13 & 25,45 & 0,73 & - & - \\
\hline \multirow[t]{2}{*}{ GDE } & Moderado & Moderado & Forte & Moderado & Moderado & - & - \\
\hline & \multicolumn{7}{|c|}{ Potássio } \\
\hline EPP & 402,00 & 317,00 & 500,00 & 1,00 & EPP & EPP & EPP \\
\hline Patamar & $1.229,00$ & 792,00 & $1.106,00$ & 739,00 & EPP & EPP & EPP \\
\hline Contribuição & 827,00 & 475,00 & 606,00 & 738,00 & EPP & EPP & EPP \\
\hline Alcance & 170,00 & 106,00 & 207,00 & 384,00 & EPP & EPP & EPP \\
\hline Modelo & Esférico & Esférico & Esférico & Esférico & EPP & EPP & EPP \\
\hline $\mathrm{R}^{2}$ & 0,98 & 0,41 & 0,37 & 0,60 & EPP & EPP & EPP \\
\hline IDE & 32,71 & 40,03 & 45,21 & 0,14 & - & - & - \\
\hline GDE & Moderado & Moderado & Moderado & Forte & - & - & - \\
\hline
\end{tabular}

${ }^{(1)} \mathrm{EPP}$, efeito pepita puro; $\mathrm{R}^{2}$, coeficiente de determinação; IDE, índice de dependência espacial; e GDE, grau da dependência espacial, de acordo com a classificação proposta por Cambardella et al. (1994). 
de $\mathrm{K}$, os valores do CDR variaram de 11,7 a $19,4 \%$. Esses resultados são indicativos de que o CDR foi um parâmetro eficiente para avaliar a similaridade dos mapas temáticos de atributos do solo e que apresenta grandes potencialidades de uso na AP. Resultados semelhantes aos obtidos no presente trabalho foram relatados anteriormente para mapas de produtividade de soja (Coelho et al., 2009) e de milho (Spezia et al., 2012).

Para os mapas de $\mathrm{P}$, o comportamento da correlação linear de Pearson (Tabela 2) foi semelhante ao verificado por meio do CDR, o que indica redução da correlação entre o mapa obtido na malha 50x50 m (referência) e os mapas interpolados das demais malhas estudadas, à
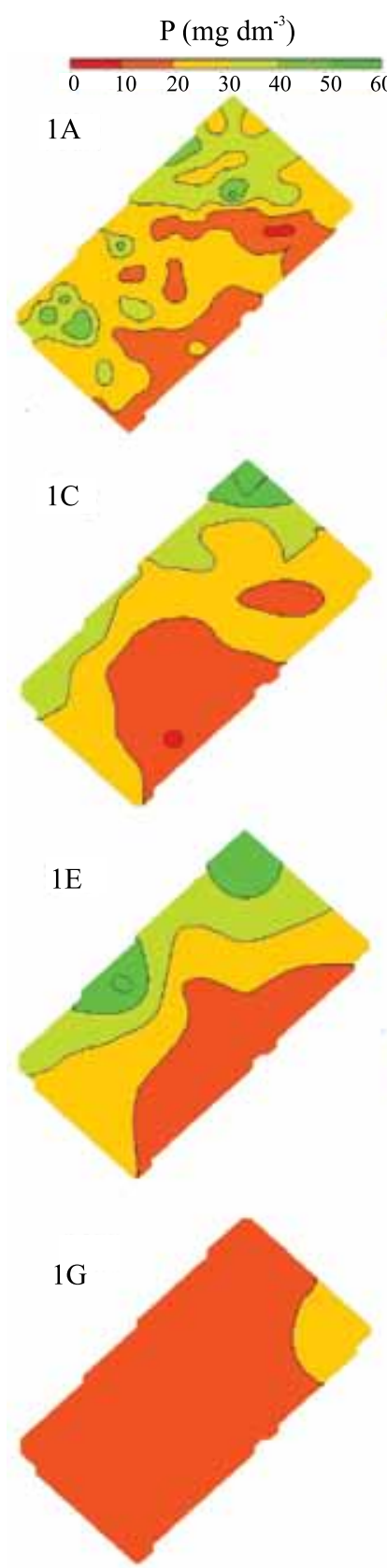
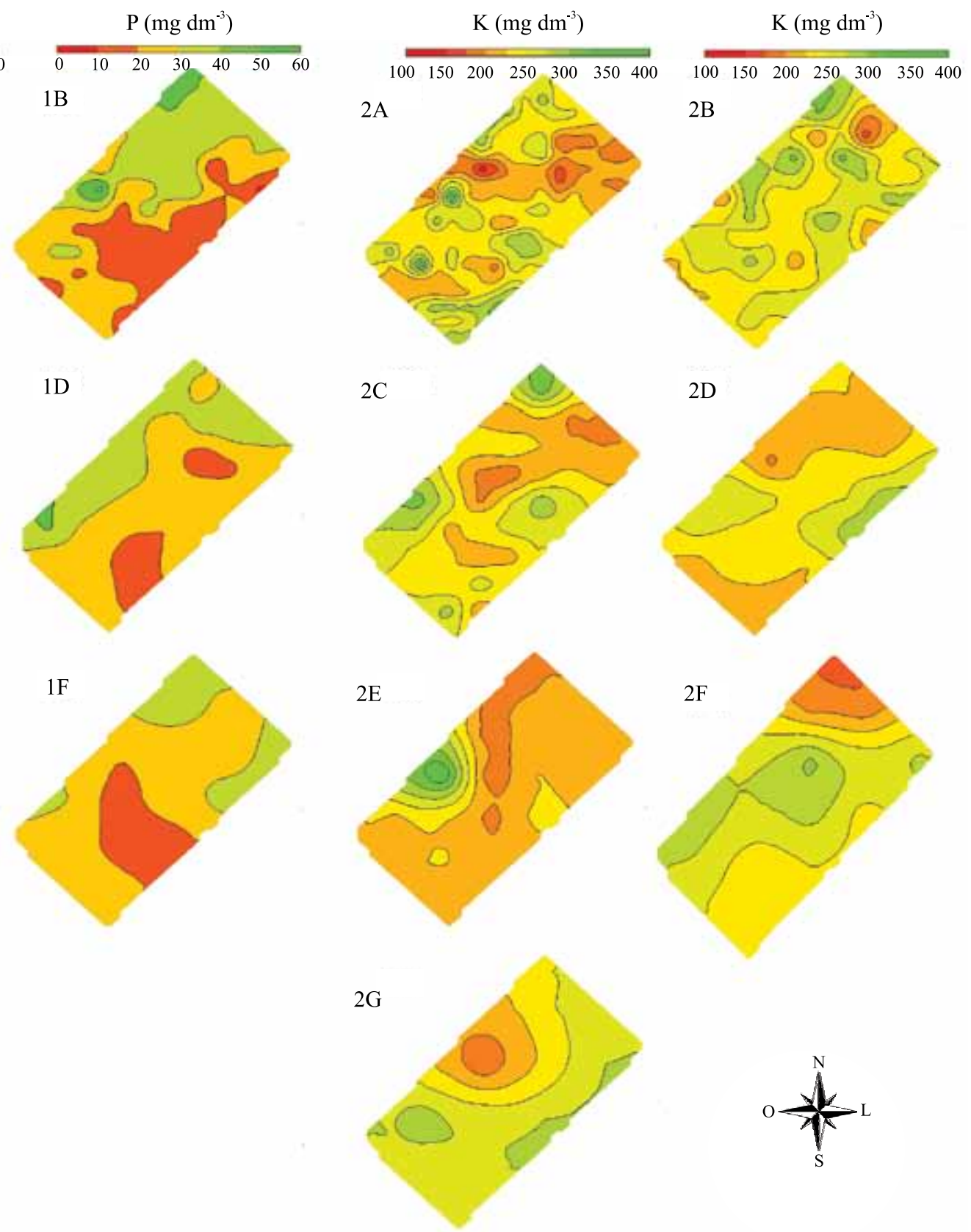

Figura 1. Espacialização dos teores de fósforo (1) e potássio (2) disponíveis, determinados com uso de diferentes dimensões de malhas amostrais, em que: A, 50x50 m; B, 75x75 m; C, 100x100 m; D, 125x125 m; E, 150x150 m; F, 175x175 m; e G, 200x200 m, em área de Latossolo Vermelho no Estado do Rio Grande do Sul. 
medida que a dimensão da malha amostral aumentou. Ou seja, quanto maior a malha amostral, maiores são os desvios e, consequentemente, os erros dos mapas temáticos gerados. Todas as malhas amostrais se correlacionaram significativamente com a malha de referência (Tabela 3), com coeficientes de correlação (r) que variaram de $\mathrm{r}=0,56$ (malha $75 \times 75 \mathrm{~m}$ ) a $\mathrm{r}=0,26$ (malha 200x200 m). No entanto, para os mapas de $\mathrm{K}$, não foi possível predizer um padrão de redução da similaridade entre os mapas temáticos a partir da análise de correlação. Isso, provavelmente, deve-se à maior dinâmica desse nutriente no solo, o que mostra

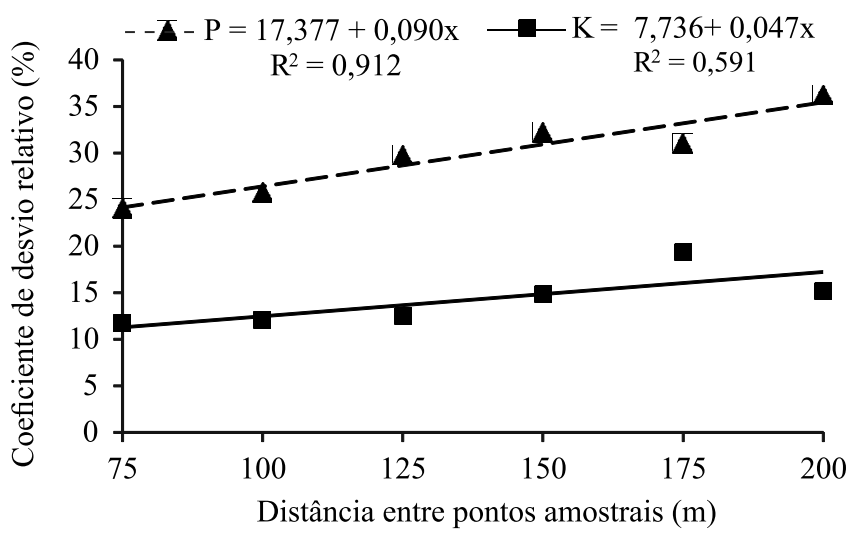

Figura 2. Coeficientes de desvio relativo entre os mapas dos teores de fósforo e potássio obtidos nas diferentes dimensões de malha amostral em área de Latossolo Vermelho no Estado do Rio Grande do Sul. um comportamento espacial menos estruturado em relação ao $\mathrm{P}$ (Tabela 2). As únicas malhas amostrais que se correlacionaram com a malha de referência (50x50 m) foram: 100x100 m, 175x175 m e 200x200 m (Tabela 3).

A dissimilaridade dos mapas temáticos de $\mathrm{P}$ e $\mathrm{K}$ ou a falta de reprodutibilidade da variabilidade espacial observada no presente trabalho deve-se, em parte, ao fato de terem sido realizadas coletas de solo independentes, para cada malha amostral, tendo-se obtido diferentes conjuntos de dados, diferentemente de outros estudos (Mallarino \& Wittry, 2004; Nanni et al., 2011; Stepien et al., 2013; Souza et al., 2014; Stamper et al., 2014), nos quais foram realizadas simulações das amostragens em malhas crescentes a partir de um mesmo conjunto de dados. Assim, os resultados de amostras de solo independentes, mesmo que coletadas próximas, estão sujeitas a importantes variações em função da variabilidade espacial do solo em curtas distâncias (Guarçoni M. et al., 2006) e às imprecisões e aos erros analíticos no laboratório.

A qualidade (acurácia) da caracterização da variabilidade espacial de $\mathrm{P}$ e $\mathrm{K}$ no solo e o consequente sucesso das aplicações corretivas em taxas variáveis de nutrientes dependem da utilização de malhas amostrais adensadas, que possibilitem coletar um número de amostras suficiente para captar as diferentes escalas de variabilidade que ocorrem na área. Dessa forma, a redução da dimensão da malha amostral deve ser preconizada em planos de amostragem de solo adotados nas áreas manejadas com AP no Estado do Rio Grande do Sul.

Tabela 3. Coeficientes de correlação linear simples de Pearson entre os mapas dos teores de fósforo (acima da diagonal) e potássio (abaixo da diagonal) disponíveis determinados com uso de diferentes dimensões de malha amostral em área de Latossolo Vermelho no Estado do Rio Grande do Sul.

\begin{tabular}{|c|c|c|c|c|c|c|c|}
\hline \multirow[t]{2}{*}{ Malha amostral } & \multicolumn{7}{|c|}{ Malha amostral (m) } \\
\hline & $50 \times 50$ & $75 \times 75$ & $100 \times 100$ & $125 \times 125$ & $150 \times 150$ & $175 \times 175$ & $200 \times 200$ \\
\hline $50 \times 50$ & & $0,56^{*}$ & $0,53 *$ & $0,47 *$ & $0,45^{*}$ & $0,32 *$ & $0,26^{*}$ \\
\hline $75 \times 75$ & 0,12 & & $0,71 *$ & $0,57 *$ & $0,72 *$ & $0,47 *$ & $0,54^{*}$ \\
\hline $100 \times 100$ & $0,34 *$ & $0,24 *$ & & $0,68 *$ & $0,74 *$ & $0,69 *$ & $0,60 *$ \\
\hline $125 \times 125$ & 0,13 & 0,10 & $0,41 *$ & & $0,78^{*}$ & $0,39 *$ & $0,60 *$ \\
\hline $150 \times 150$ & 0,03 & $0,16^{*}$ & $0,27 *$ & $0,32 *$ & & $0,37^{*}$ & $0,56^{*}$ \\
\hline $175 \times 175$ & $0,20 *$ & 0,12 & $-0,05$ & $0,40^{*}$ & $0,34 *$ & & $0,55^{*}$ \\
\hline $200 \times 200$ & $0,19 *$ & $-0,22 *$ & $0,27 *$ & $0,45^{*}$ & $-0,15$ & $-0,18^{*}$ & \\
\hline
\end{tabular}

*Significativo pela correlação linear de Pearson, a 5\% de probabilidade. $n=164$ 


\section{Conclusões}

1. A redução da dimensão da malha amostral aumenta a acurácia na caracterização da variabilidade espacial de $\mathrm{P}$ e $\mathrm{K}$ por meio de mapas temáticos.

2. O uso de malhas amostrais menores ou iguais a 100x100 m, com uma ou mais amostras por hectare, é indicado para caracterizar a variabilidade espacial de $\mathrm{P}$ e K em áreas sob agricultura de precisão no Estado do Rio Grande do Sul.

3. Os mapas de fertilidade do solo tornam-se mais dissimilares à medida que aumenta a dimensão de malha amostral, e esses desvios são mais significativos para o $\mathrm{P}$ do que para o $\mathrm{K}$.

4. O coeficiente de desvios relativos é um parâmetro eficiente para avaliar a similaridade de mapas temáticos de atributos de solo e pode ser utilizado na agricultura de precisão.

\section{Agradecimentos}

À Coordenação de Aperfeiçoamento de Pessoal de Nível Superior (Capes), pela concessão de bolsa.

\section{Referências}

CAMBARDELLA, C.A.; MOORMAN, T.B.; NOVAK, J.M.; PARKIN, T.B.; KARLEN, D.L.; TURCO, R.F.; KONOPKA, A.E. Field-scale variability of soil properties in central Iowa soils. Soil Science Society of America Journal, v.58, p.1501-1511, 1994. DOI: 10.2136/sssaj1994.03615995005800050033x.

CHERUBIN, M.R.; SANTI, A.L.; BASSO, C.J.; EITELWEIN, M.T.; VIAN, A.L. Caracterização e estratégias de manejo da variabilidade espacial dos atributos químicos do solo utilizando a análise dos componentes principais. Enciclopédia Biosfera, v.7, p.196-210, 2011.

CHERUBIN, M.R.; SANTI,A.L.; EITELWEIN, M.T.; MENEGOL, D.R.; DA ROS, C.O.; PIAS, O.H. de C.; BERGHETTI, J. Eficiência de malhas amostrais utilizadas na caracterização da variabilidade espacial de fósforo e potássio. Ciência Rural, v.44, p.425-432, 2014. DOI: $10.1590 / \mathrm{S} 0103-84782014000300007$.

COELHO, E.C.; SOUZA, E.G. de; URIBE-OPAZO, M.A.; PINHEIRO NETO, R. Influência da densidade amostral e do tipo de interpolador na elaboração de mapas temáticos. Acta Scientiarum. Agronomy, v.31, p.165-174, 2009. DOI: 10.4025/ actasciagron.v31i1.6645.

CORÁ, J.E.; BERALDO, J.M.G. Variabilidade espacial de atributos do solo antes e após calagem e fosfatagem em doses variadas na cultura de cana-de-açúcar. Engenharia Agrícola, v.26, p.374-387, 2006. DOI: 10.1590/S0100-69162006000200005.

ERNANI, P.R.; ALMEIDA, J.A. de; SANTOS, F.C. dos. Potássio. In: NOVAIS, R.F.; ALVAREZ V., V.H.; BARROS, N.F. de;
FONTES, R.L.F.; CANTARUTTI, R.B.; NEVES, J.C.L. (Ed.). Fertilidade do solo. Viçosa: Sociedade Brasileira de Ciência do Solo, 2007. p.551-594.

GUARÇONI M., A.; ALVAREZ V., V.H.; NOVAIS, R.F.; CANTARUTTI, R.B.; LEITE, H.G.; FREIRE, F.M. Definição da dimensão do indivíduo solo e determinação do número de amostras simples necessário à sua representação. Revista Brasileira de Ciência do Solo, v.30, p.943-954, 2006. DOI: 10.1590/ S0100-06832006000600004.

KERRY, R.; OLIVER, M.A. Average variograms to guide soil sampling. International Journal of Applied Earth Observation and Geoinformation, v.5, p.307-325, 2004. DOI: 10.1016/j. jag.2004.07.005.

LANDIM, P.M.B. Sobre geoestatística e mapas. Terræ Didatica, v.2, p.19-33, 2006.

MAGRI, A.; VAN ES, H.M.; GLOS, M.A.; COX, W.J. Soil test, aerial image and yield data as inputs for site-specific fertility and hybrid management under maize. Precision Agriculture, v.6, p.87-110, 2005. DOI: 10.1007/s11119-004-0687-7.

MALLARINO, A.P.; WITTRY, D.J. Efficacy of grid and zone soil sampling approaches for site-specific assessment of phosphorus, potassium, pH, and organic matter. Precision Agriculture, v.5, p.131-144, 2004. DOI: 10.1023/B:PRAG.0000022358.24102.1b.

MALUF, J.R.T. Nova classificação climática do Estado do Rio Grande do Sul. Revista Brasileira de Agrometeorologia, v.8, p.141-150, 2000.

MANUAL de adubação e de calagem para os Estados do Rio Grande do Sul e Santa Catarina. 10.ed. Porto Alegre: Sociedade Brasileira de Ciência do Solo, Núcleo Regional Sul, 2004. 400p.

MONTANARI, R.; SOUZA, G.S.A.; PEREIRA, G.T.; MARQUES JUNIOR, J.; SIQUEIRA, D.S.; SIQUEIRA, G.M. The use of scaled semivariograms to plan soil sampling in sugarcane fields. Precision Agriculture, v.13, p.542-552, 2012. DOI: 10.1007/ s11119-012-9265-6.

NANNI, M.R.; POVH, F.P.; DAMATTÊ, J.A.M.; OLIVEIRA, R.B. de; CHICATI, M.L.; CEZAR, E. Optimum size in grid soil sampling for variable rate application in site-specific management. Scientia Agricola, v.68, p.386-392, 2011. DOI: 10.1590/ S0103-90162011000300017.

SANTI, A.L.; AMADO, T.J.C.; CHERUBIN, M.R.; MARTIN, T.N.; PIRES, J.L.; DELLA FLORA, L.P.; BASSO, C.J. Análise de componentes principais de atributos químicos e físicos do solo limitantes à produtividade de grãos. Pesquisa Agropecuária Brasileira, v.47, p.1346-1357, 2012. DOI: 10.1590/ S0100-204X2012000900020.

SANTOS, H.G. dos; JACOMINE, P.K.T.; ANJOS, L.H.C. dos; OLIVEIRA, V.A. de; LUMBRERAS, J.F.; COELHO, M.R.; ALMEIDA, J.A.; CUNHA, T.J.F.; OLIVEIRA, J.B. de (Ed.). Sistema brasileiro de classificação de solos. 3.ed. rev. ampl. Brasília: Embrapa, 2013. 353p.

SOUZA, Z.M. de; SOUZA, G.S. de; MARQUES JÚNIOR, J.; PEREIRA, G.T. Número de amostras na análise geoestatística e na krigagem de mapas de atributos do solo. Ciência Rural, v.44, p.261-268, 2014. DOI: 10.1590/S0103-84782014000200011. 
SPEZIA, G.R.; SOUZA, E.G. de; NOBREGA, L.H.P.; URIBE-OPAZO, M.A.; MILAN, M.; BAZZI, C.L. Model to estimate the sampling density for establishment of yield mapping. Revista Brasileira de Engenharia Agrícola e Ambiental, v.16, p.449-457, 2012. DOI: 10.1590/ S1415-43662012000400016.

STAMPER, D.J.; AGOURIDIS, C.T.; EDWARDS, D.R.; PURSCHWITZ, M.A. Effect of soil sampling density and landscape features on soil test phosphorus. Applied Engineering in Agriculture, v.30, p.773-781, 2014.

STEPIEN, M.; GOZDOWSKI, D.; SAMBORSKI, S. A case study on the estimation accuracy of soil properties and fertilizer rates for different soil-sampling grids. Journal of Plant Nutrition and Soil Science, v.176, p.57-68, 2013. DOI: 10.1002/jpln.201100422.
VIEIRA, S.R.; MILLETE, J.; TOPP, G.C.; REYNOLDS, W.R. Handbook for geostatistical analysis of variability in soil and climate data. In: ALVAREZ V., V.H.; SCHAEFER, C.E.G.R.; BARROS, N.F. de; MELLO, J.W.V. de; COSTA, L.M. da (Ed.). Tópicos em ciência do solo. Viçosa: Ed. da UFV, 2002. v.2, p.1-46. WEBSTER, R.; LARK, M. Field sampling for environmental science and management. London: Routledge, 2012. 200p.

WEBSTER, R.; OLIVER, M.A. Geostatistics for environmental scientists. $2^{\text {nd }}$ ed. Chichester: J. Wiley, 2007. 330p. DOI: 10.1002/9780470517277.

WILDING, L.P.; DREES, L.R. Spatial variability and pedology. In: WILDING, L.P.; SMECK, N.E.; HALL, G.F. (Ed.). Pedogenesis and soil taxonomy. I. Concepts and Interactions. Amsterdam: Elsevier, 1983. p.83-116. DOI: 10.1016/S0166-2481(08)70599-3.

Recebido em 23 de agosto de 2013 e aprovado em 27 de janeiro de 2015 